\title{
Autofocused Compressive SAR Imaging based on the Alternating Direction Method of Multipliers
}

\author{
Alper Güngör \\ ASELSAN Research Center, \\ ASELSAN Inc., Ankara, Turkey \\ alpergungor@aselsan.com.tr
}

\author{
Müjdat Çetin \\ Faculty of Engineering \\ and Natural Sciences, \\ Sabancı University, Istanbul, Turkey, \\ mcetin@sabanciuniv.edu
}

\author{
H. Emre Güven \\ ASELSAN Research Center \\ ASELSAN Inc., Ankara, Turkey \\ heguven@aselsan.com.tr
}

\begin{abstract}
We present an alternating direction method of multipliers (ADMM) based autofocused Synthetic Aperture Radar (SAR) imaging method in the presence of unknown 1-D phase errors in the phase history domain, with undersampled measurements. We formulate the problem as one of joint image formation and phase error estimation. We assume sparsity of strong scatterers in the image domain, and as such use sparsity priors for reconstruction. The algorithm uses $\ell_{p}$-norm minimization $(p \leq 1)$ [8] with an improvement by integrating the phase error updates within the alternating direction method of multipliers (ADMM) steps to correct the unknown 1-D phase error. We present experimental results comparing our proposed algorithm with a coordinate descent based algorithm in terms of convergence speed and reconstruction quality.
\end{abstract}

\section{INTRODUCTION}

Phase errors in Fourier domain must be corrected before forming synthetic aperture radar (SAR) images. The phase errors occur due to non-ideal propagation media or navigation errors. The errors degrade image quality. There are several algorithms that can estimate and correct the phase error, however these algorithms have not been proven to work well in the context of compressed sensing. While sparsity-driven autofocused SAR imaging has been proposed before [1], [2], [3], [4], [5], computationally efficient algorithms for practical use remains a challenge. Our contribution here is an improved and computationally efficient alternating direction method of multipliers (ADMM) based algorithm for autofocused compressive SAR imaging.

ADMM is an augmented Lagrangian method based algorithm that solves a given problem by dividing it into two easier sub-problems and augmenting each sub-problem with a Lagrangian term for fast convergence. The resulting algorithm converges under mild conditions [6]. The method has been shown to be useful and fast for SAR formulation [7], [8].

In this study, we tackle the problem of reconstructing synthetic aperture radar (SAR) images from compressive measurements, while estimating 1-D phase error in azimuth direction in the phase history domain. We solve a problem similar to [1] using ADMM framework. The augmented Lagrangian helps speed up the convergence of the algorithm. We demonstrate the effectiveness of the algorithm on two datasets: a synthetic [9], and an experimental [10]. We demonstrate the algorithm on the experimental data collected with SARPER ${ }^{\mathrm{TM}}$, airborn SAR system developed by ASELSAN [10]. We show the im- provements on the image quality and the speed of convergence over a previous proposed framework in terms of RMSE [1].

\section{BACKGROUND}

\section{A. Observation Model}

A linear observation model can be considered as SAR observation model in relating the image to collected data vector. Although we will refer to matrix notation, the matrices are not formed explicitly. We assume that the data lies in phase history domain, and the forward model is simply the selection of some elements in Fourier transformation of the reflectivity field, and the operations including the forward model are carried out using fast Fourier transform (FFT) algorithm. Let B $\in \mathbb{C}^{M x N}$ with $M<N$ denote the forward operator, $\mathbf{y} \in \mathbb{C}^{M}$ denote the data vector, and $\mathbf{x} \in \mathbb{C}^{N}$ denote the vector formed by concatenating image into vector form. Let the unknown 1-D phase error term be denoted by $\phi \in \Re^{n}, n$ being the number of samples in the azimuth direction. Let us define $\mathbf{B}$ as forward model:

$$
\mathbf{B}=\mathbf{M U}
$$

where $\mathbf{M} \in \Re^{M x N}$ is the element selection matrix, and $\mathbf{U}$ is the 2-D Fourier transformation matrix. Let us now define corrected data as:

$$
\mathbf{y}(\boldsymbol{\phi})=\mathbf{y} \cdot \exp \{j \phi\}
$$

where the multiplication is defined as multiplying each element $\mathbf{y}_{i}$ of $\mathbf{y}$ with the corresponding phase correction element $\phi[i]$. Here multiple elements of the data vector corresponds to the same element in $\phi$ depending on the row that $\mathbf{y}$ lies in. The measurement vector $\mathbf{y}(\phi)$ is given by the model:

$$
\mathbf{y}(\phi)=\mathbf{B x}+\boldsymbol{\eta},
$$

where $\boldsymbol{\eta} \in \mathbb{C}^{M}$ denotes the additive noise vector.

\section{B. Sparsity Driven Autofocus (SDA)}

The outlined problem of 1-D autofocus is a problem of estimation of the phase error vector $\phi$. There are several sparsity driven approaches in the literature that attack this 
problem [2], [3], [11], [4]. The methods usually solve a sparsity enhancing optimization problem by minimizing the functional:

$$
J(\mathbf{f}, \phi)=\|y-\mathbf{B}(\phi) \mathbf{f}\|_{2}^{2}+\lambda\|\mathbf{f}\|_{1}
$$

where $\mathbf{B}(\phi)$ denotes phase corrected model. Here, note that the problems Eq. (2) and Eq. (4) are equivalent.

The proposed method in [2] minimizes objective function $J(\mathbf{f}, \phi)$ over both $\mathbf{f}$, and $\phi$. The algorithm separates for two variables, then applies coordinate descent to solve it. It uses conjugate gradient for the minimizing with respect to $f$, and analytical solution for minimizing with respect to $\phi$. We only deal with 1-D phase errors, however, [2] includes solving the proposed problem for 1-D, 2-D separable, and 2-D nonseparable cases. For this reason, we only give the analytical solution for 1-D phase error as:

$$
\phi_{1-D}^{(k+1)}[m]=-\arctan \left(\frac{\operatorname{Im}\left\{\mathbf{f}^{(k+1)^{H}} \mathbf{B}_{m}\left(\phi^{(k)}\right)^{H} \mathbf{y}_{m}\right\}}{\operatorname{Re}\left\{\mathbf{f}^{(k+1)^{H}} \mathbf{B}_{m}\left(\phi^{(k)}\right)^{H} \mathbf{y}_{m}\right\}}\right)
$$

$$
\mathbf{B}_{m}\left(\boldsymbol{\phi}_{1-D}^{(k+1)}[m]\right)=\exp \left(j \phi_{1-D}^{(k+1)}[m]\right) \mathbf{B}_{m}\left(\boldsymbol{\phi}^{(k)}\right) .
$$

\section{Autofocusing ADMM}

In this section we propose recovering SAR images by solving an optimization problem with a computationally efficient algorithm. A previous version of the algorithm solves the problem using a coordinate descent structure with a conjugate gradient or ADMM to update the image estimate in each outer step of the iterations [1], [2]. Here, we propose an ADMM based algorithm that converges quickly using the power of ADMM, within a linear model [1], [2], [7]. We assume that the scene is sparse, and the unknown phase error is 1-dimensional. Hence, we solve the following problem [1], [2]:

$$
\begin{array}{cc}
\underset{\mathbf{x}, \boldsymbol{\phi}}{\operatorname{minimize}} & f(\mathbf{x}) \\
\text { subject to } & \|\mathbf{B} \mathbf{x}-\mathbf{y} \cdot \exp \{j \phi\}\|_{2} \leq \epsilon
\end{array},
$$

where $f(\cdot)$ denotes the sparsity prior such as $\ell_{p}$-norm with $p \leq$ 1 , B denotes the forward model of masked Fourier transform, $\mathbf{y}$ denotes received signal, $\phi$ denotes the unknown phase, and $\epsilon$ denotes the bound on data-fidelity. In order to find an optimal $\mathbf{x}$ in (7), we use ADMM, which in its most general form solves problems of the type:

$$
\begin{array}{lc}
\underset{\mathbf{x}}{\operatorname{minimize}} & f_{1}(\mathbf{x})+f_{2}(\mathbf{z}) \\
\text { subject to } & \mathbf{G x}+\mathbf{Q z}-\mathbf{m}=0
\end{array} .
$$

In (8) we let

$$
\begin{aligned}
& \mathbf{z}=\left[\begin{array}{c}
\mathbf{z}^{(\mathbf{1})} \\
\mathbf{z}^{(\mathbf{2})} \\
\boldsymbol{\phi}
\end{array}\right], \quad \mathbf{G}=\left[\begin{array}{l}
\mathbf{I} \\
\mathbf{B}
\end{array}\right], \\
& \mathbf{Q}=\left[\begin{array}{ccc}
-\mathbf{I} & 0 & 0 \\
0 & -\mathbf{I} & 0
\end{array}\right], \quad \mathbf{m}=0, \quad f_{1}(\mathbf{x})=0
\end{aligned}
$$

and

$$
f_{2}(\mathbf{z})=f\left(\mathbf{z}^{(\mathbf{1})}\right)+\iota_{E(\epsilon, \mathbf{I}, \mathbf{y} \cdot \exp \{j \phi\})}\left(\mathbf{z}^{(\mathbf{2})}\right),
$$

where $\iota_{E(\epsilon, \mathbf{I}, \mathbf{y} \cdot \exp \{j \phi\})}(\mathbf{x})$ accounts for the indicator function associated with the data fidelity constraint $\|\mathbf{B} \mathbf{x}-\mathbf{y}\|_{2} \leq \epsilon$ as:

$$
\begin{gathered}
E(\epsilon, \mathbf{A}, \mathbf{y})=\left\{\mathbf{x} \in \mathbf{C}^{N}:\|\mathbf{A} \mathbf{x}-\mathbf{y}\|_{2} \leq \epsilon\right\}, \\
\iota_{S}(\mathbf{s})= \begin{cases}0, & \text { if } \mathbf{s} \in S \\
+\infty, & \text { if } \mathbf{s} \notin S\end{cases}
\end{gathered}
$$

This setting ensures that $\mathbf{G x}=\mathbf{z}$, and consequently: $\mathbf{x}=$ $\mathbf{z}^{(\mathbf{1})}, \mathbf{B x}=\mathbf{z}^{(\mathbf{2})}$ and that there is no constraint on $\phi$. Note that $\phi \in R^{n_{1}}$, and not $R^{n_{1} \times n_{2}}$, where $n_{1}$ and $n_{2}$ denote the size of the reconstructed image in two dimensions. $\mathbf{y} \cdot \exp \{j \phi\}$ denotes the phase correction step of the data. $i$-th element of the result is calculated as $\mathbf{y}[i] \exp \{j \phi[m]\}$, where $m$ is the azimuth index corresponding to data point $\mathbf{y}[i]$.

ADMM consists of the following iterations:

$$
\begin{aligned}
& \mathbf{x}_{n+1}=\arg \min _{\mathbf{x}} f_{1}(\mathbf{x})+\frac{\mu}{2}\left\|\left(\mathbf{G x}+\mathbf{Q} \mathbf{z}_{n}+\mathbf{d}_{n}\right)\right\|_{2}^{2} \\
& \mathbf{z}_{n+1}=\arg \min _{\mathbf{z}} f_{2}(\mathbf{z})+\frac{\mu}{2}\left\|\left(\mathbf{G} \mathbf{x}_{\mathbf{n}+\mathbf{1}}+\mathbf{Q z}+\mathbf{d}_{n}\right)\right\|_{2}^{2} \\
& \mathbf{d}_{n+1}=\mathbf{d}_{n}+\mathbf{G} \mathbf{x}_{\mathbf{n}+\mathbf{1}}+\mathbf{Q} \mathbf{z}_{n+1}
\end{aligned}
$$

Then, $\mathbf{x}$-update step is a simple least squares solution, and an efficient solution has been previously provided in the SAR imaging context [7], using two FFTs per iteration. The zupdate step consists of two separable equations as in [12]:

$$
\begin{aligned}
& \mathbf{z}_{n+1}^{(1)}=\arg \min _{\mathbf{z}^{(1)}} f\left(\mathbf{z}^{(\mathbf{1})}\right)+\frac{\mu}{2}\left\|\mathbf{z}^{(\mathbf{1})}-\left(\mathbf{x}-\mathbf{d}_{n}\right)\right\|_{2}^{2} \\
& \mathbf{z}_{k+1}^{(2)}, \phi_{k+1}=\arg \min _{\mathbf{z}^{(\mathbf{2})}, \boldsymbol{\phi}} \iota_{E(\epsilon, \mathbf{I}, \mathbf{y} \cdot \exp \{j \phi\})}\left(\mathbf{z}^{(\mathbf{2})}\right) \\
& +\frac{\mu}{2}\left\|\mathbf{B} \mathbf{x}_{k+1}-\mathbf{z}^{(2)}-\mathbf{d}_{k}^{(2)}\right\|_{2}^{2},
\end{aligned}
$$

The update step related to (16) is known as the Moreau proximal mapping [12] of $f$. In this study, we use $\ell_{p}$-norm as the objective function, for which an approximation to the associated proximal mapping has been previously defined [8].

Next we reformulate (17) by putting the problem into the constrained form as follows:

$$
\begin{array}{r}
\mathbf{z}_{k+1}^{(2)}=\arg \min _{\mathbf{z}^{(2)}, \boldsymbol{\phi}}\left\|\mathbf{B} \mathbf{x}_{k+1}-\mathbf{z}^{(2)}-\mathbf{d}_{k}^{(2)}\right\|_{2}^{2} \\
\text { subject to }\left\|\mathbf{z}^{(2)}-\mathbf{y} \cdot \exp \{j \phi\}\right\|_{2} \leq \epsilon .
\end{array}
$$

The solution can be found in two steps. Let $\mathbf{y}_{k}=\mathbf{y}$. $\exp \left\{j \phi_{k}\right\}$, and $\mathbf{s}=\mathbf{B x}_{k+1}-\mathbf{d}_{k}^{(2)}$. Then for fixed $\boldsymbol{\phi}$, the solution to the equation is a simple projection onto the hypersphere, given by:

$$
\boldsymbol{\Psi}_{\iota_{E\left(\epsilon, \mathbf{I}, \mathbf{y}_{k}\right)}}(\mathbf{s})=\left\{\begin{array}{ll}
\mathbf{s}, & \text { if }\left\|\mathbf{s}-\mathbf{y}_{k}\right\|_{2} \leq \epsilon \\
\mathbf{y}_{k}+\epsilon \frac{\left(\mathbf{s}-\mathbf{y}_{k}\right)}{\left\|\mathbf{s}-\mathbf{y}_{k}\right\|_{2}}, & \text { if }\left\|\mathbf{s}-\mathbf{y}_{k}\right\|_{2}>\epsilon
\end{array} .\right.
$$

Hence, the solution always lies on the line between $\mathbf{y}_{k}$ and $\mathbf{s}$. Choosing $\phi$ to minimize the Euclidian distance between $\mathbf{y}_{k}$ and $\mathbf{s}$, we obtain an analytical solution for $\phi$ in (17) through

$$
\phi_{k+1}=\arg \min _{\boldsymbol{\phi}}\left\|\mathbf{y} \cdot \exp \left\{j \phi_{k}\right\}-\mathbf{s}\right\|_{2}^{2},
$$


the solution of which can be derived as [1], [2]:

$$
\begin{gathered}
\phi[m]=-\arctan 2\left(\operatorname{Im}\left\{\left(\mathbf{B} \mathbf{x}_{k+1}-\mathbf{d}_{k}^{(2)}\right)_{m}^{H} \mathbf{y}_{m}\right\},\right. \\
\left.\operatorname{Re}\left\{\left(\mathbf{B x}_{k+1}-\mathbf{d}_{k}^{(2)}\right)_{m}^{H} \mathbf{y}_{m}\right\}\right)
\end{gathered}
$$

where $\arctan 2$ denotes the four-quadrant arctangent, and $m$ denotes the azimuth index. Consequently, $\mathbf{z}^{(\mathbf{2})}$ can be calculated using (19) with a simple projection.

The resulting method is given in Algorithm 1.

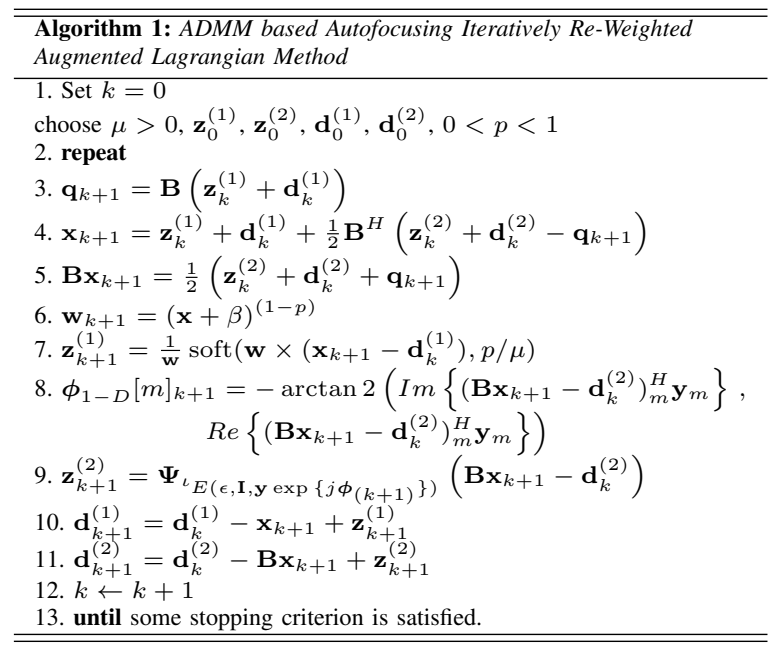

While the guarantees for convergence to global minimum in the convex case [6] do not apply here, we observe that the algorithm performs successfully, even for smaller $p$ values such as $p=0.1$, as studied in the next section.

\section{RESULTS}

We implemented the algorithm in MATLAB and ran the reconstruction routines on a workstation with dual Intel Xeon E5-2650 v2 and 64 GB of RAM. The values of all dual variables $\left(\mathbf{z}^{(\mathbf{1})}, \mathbf{z}^{(\mathbf{2})}, \mathbf{d}^{(1)}, \mathbf{d}^{(2)}\right)$ were initialized to zero.

First, we show the results on a simulated dataset. For this purpose, we purposely add 1-D phase error in azimuth direction on a backhoe image [9]. Then, we subsample the image in the Fourier domain to $39 \%$ of full-data using a random binary mask. We consider two reduced data scenarios (1) rectangular sub-sampling which models limitations in signal bandwidth or azimuth aperture length, and (2) random subsampling to model an irregular data limitation scenario. We also study the performance of the method with a rectangular mask by subsampling the image at a rate of $25 \%$ with respect to the full data in the Fourier space centered around the origin. We run the proposed autofocused ADMM algorithm and AIRWALM [1] using different $p$ values. Figure 1 and Fig. 2 show the change of error in the estimated phase values with respect to the number of iterations for both algorithms, with different values of $p$. Coordinate descent with smaller values of $p$ does not converge, which is the reason for the absence of the corresponding curves in Fig. 1 and Fig. 2. We note that the smaller values of $p$ yield better performance (smaller phase error) for both algorithms, while convergence of coordinate descent is compromised with smaller $p$. Figure 3 shows the reference image with phase error (Fig. 3a), undersampled image with a rectangular mask

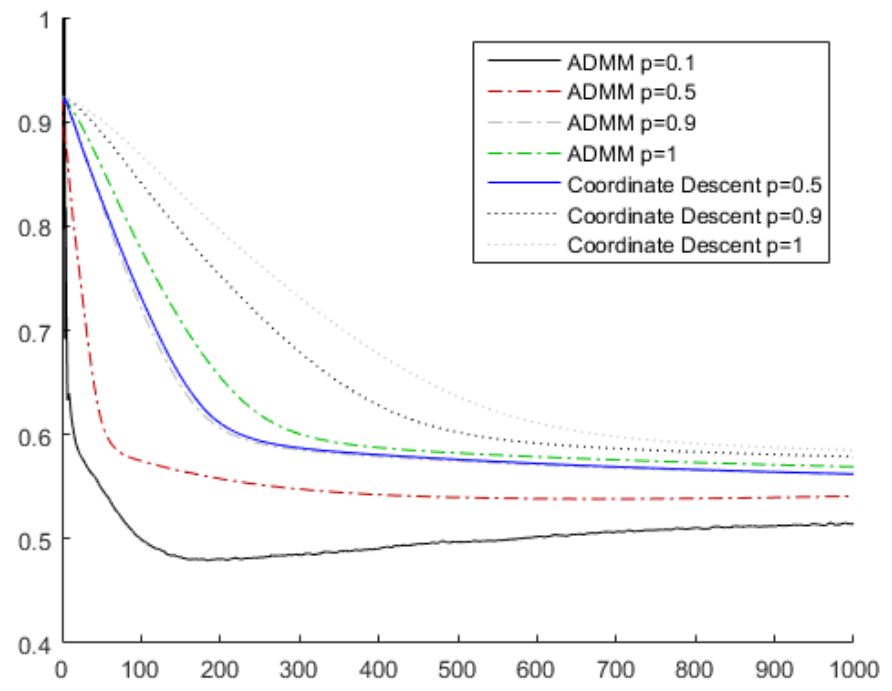

Fig. 1. RMSE in Phase Error estimation in Radians versus iteration for Autofocusing ADMM and AIRWALM(Coordinate Descent) with different $p$ values using a random mask for data reduction in the Fourier space

(Fig. 3b), and reconstruction results for $p=1$ and $p=0.1$ (Fig. 3c-d). As it can be seen, reconstruction images can successfully estimate and cancel undersampling and phase error effects.

Next, we show the results on real data obtained with SARPER $^{\text {TM }}$ - airborne SAR system developed by ASELSAN [10]. Again, just as in the previous experiment, we subsample the image to $39 \%$ using a random binary mask. Then, we run the proposed algorithm with different values of $p$. Figure 4 shows the reconstructions of the proposed method for different $p$-values at the end of 200 iterations. Before reconstruction, the image has both phase error and undersampling artifacts as it can be seen in Fig. 4a and Fig. 4b. The reconstruction images show an improvement with no artifact present. As $p$ decreases, point scatterers are enhanced even further, and hence the dark spots on the figures constitute visibly higher contrast.

\section{CONCLUSION}

In this study, we proposed an augmented Lagrangian method based algorithm for autofocused compressive SAR imaging. We derived the method based on alternating direction method of multipliers, and experimentally studied its performance on simulated and real datasets. We compared the algorithm to a previous approach based on coordinate descent and observed better performance in terms of the number of iterations as well as phase-error and focusing ability.

\section{REFERENCES}

[1] A. Gungor, M. Cetin, and H. E. Guven "An augmented Lagrangian method for autofocused Compressed SAR Imaging," Compressed Sensing Theory and its Applications to Radar, Sonar and Remote Sensing (CoSeRa) 2015, June 2015

[2] N. O. Onhon, M. Cetin: A Sparsity-Driven Approach for Joint SAR Imaging and Phase Error Correction, IEEE Trans. Image Processing, vol. 21, no. 4, pp. 2075-2088, April 2012.

[3] K-H. Liu, A. Wiesel, D. C. Munson Jr: Synthetic Aperture Radar Autofocus via Semidefinite Relaxation, IEEE Trans. Image Processing, vol. 22, no. 6, pp. 2317-2326, June 2013. 


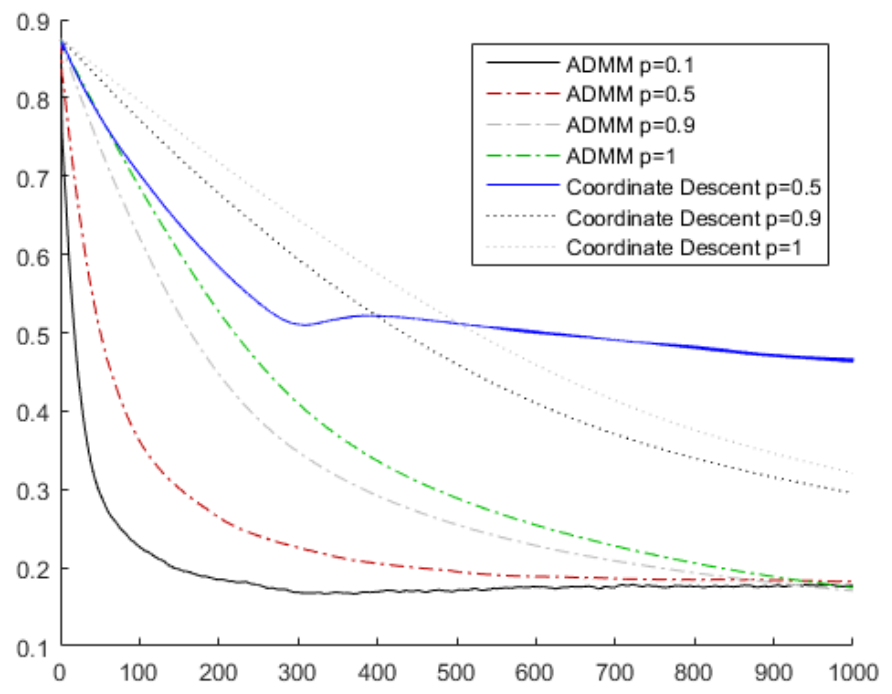

Fig. 2. RMSE in Phase Error estimation in Radians versus iteration for Autofocusing ADMM and AIRWALM with different $p$ values using a rectangular mask in the Fourier space.
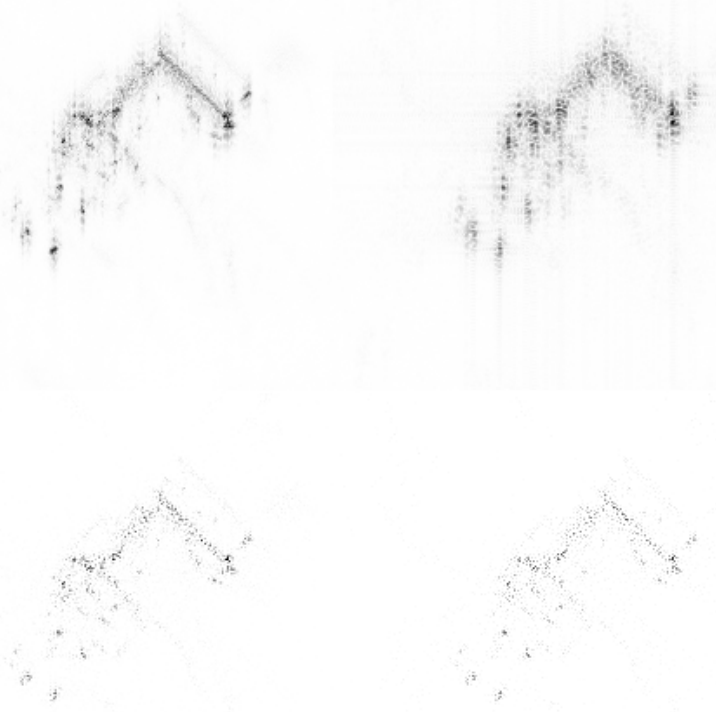

Fig. 3. a. Reference Backhoe image with 1-D phase error (upper-left), b. Zero-filled in Fourier domain reconstruction image (upper-right), c. Image reconstructed with autofocusing ADMM $\ell_{1}$ (lower-left), d. Image reconstructed with autofocusing $\mathrm{ADMM} \ell_{0.1}$ (lower-right)

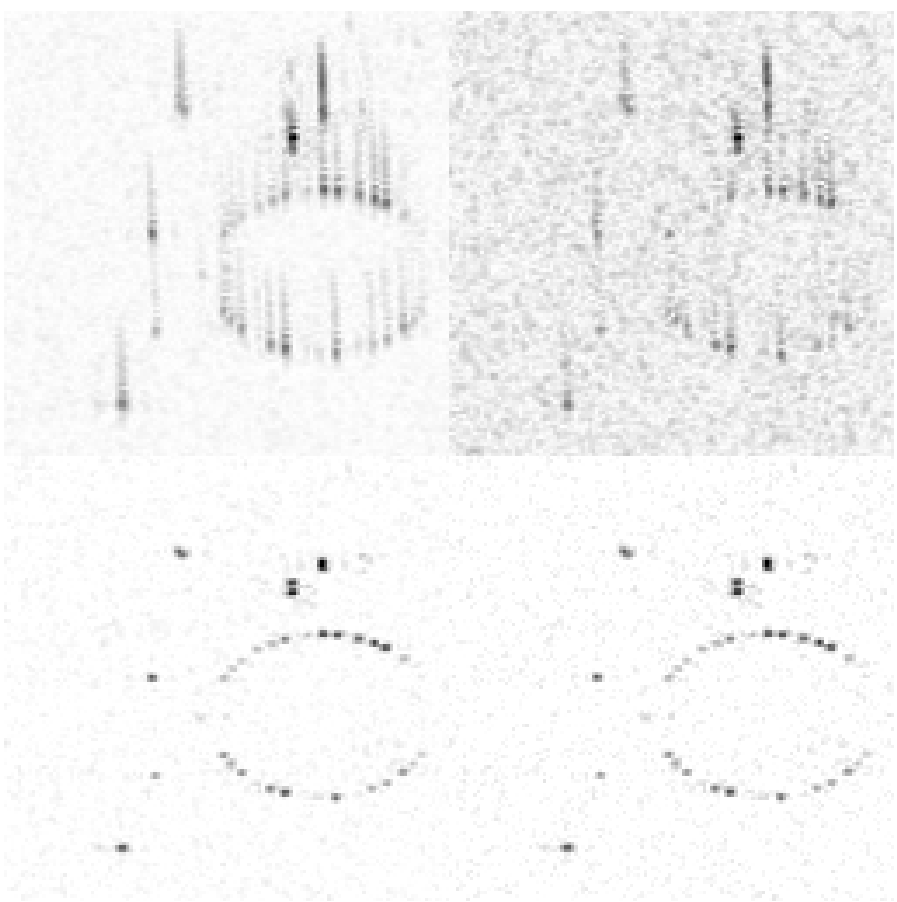

Fig. 4. a. Reference SARPER ${ }^{\mathrm{TM}}$ image (upper-left), b. Zero-filled in Fourier domain reconstruction image (upper-right), c. Image reconstructed with autofocusing $\mathrm{ADMM} \ell_{1}$ (lower-left), d. Image reconstructed with autofocusing ADMM $\ell_{0.1}$ (lower-right)

[4] J. H. G. Ender, "Autofocusing ISAR images via sparse representation," in 9th European Conference on Synthetic Aperture Radar (EUSAR 2012). EU, 2012.

[5] S. Kelly, M. Yaghoobi, M. Davies, "Sparsity-based autofocus for undersampled synthetic aperture radar," in IEEE Trans. Aeurospace and Electronic Systems, vol. 50, no. 2, pp. 972-986, April 2014.

[6] S. Boyd, N. Parikh, E. Chu, B. Peleato, and J. Eckstein, "Distributed optimization and statistical learning via the alternating direction method of multipliers," Foundations and Trends in Machine Learning, vol. 3, no. 1, pp. 1-122, 2011.

[7] H. E. Guven, A. Gungor and M. Cetin, An Augmented Lagrangian method for Complex-Valued Compressed SAR Imaging, IEEE Trans. Computational Imaging, vol. 2, no. 3, pp. 235-250, September 2016.

[8] H. E. Guven, A. Gungor and M. Cetin, "An augmented Lagrangian method for image reconstruction with multiple features," International Conference on Image Processing (ICIP 2015), September 2015.

[9] Backhoe Data Sample \& Visual-D Challenge Problem available through the Air Force Research Laboratory Sensor Data Management System Web Page: www.sdms.afrl.af.mil/main.htm.

[10] ASELSAN website for $\mathrm{SARPER}^{\mathrm{TM}}$ : http://www.aselsan.com.tr/enus/capabilities/radar-systems/

[11] S. Ugur, O. Arikan, A. C. Gurbuz: SAR image reconstruction by expectation maximization based matching pursuit, Digital Signal Processing, vol. 37, pp. 75-84, February 2015.

[12] M. Afonso, J. M. Bioucas-Dias, M. A. T. Figueiredo, An Augmented Lagrangian approach to the constrained optimization formulation of imaging inverse problems, IEEE Trans. Image Processing, vol. 20, no. 3, pp. 681-695, March 2011. 